\title{
MK-2206 window of opportunity study in breast cancer
}

\author{
Argun Akcakanat ${ }^{1}$, Funda Meric-Bernstam ${ }^{1,2,3,4}$ \\ ${ }^{1}$ Department of Investigational Cancer Therapeutics, ${ }^{2}$ Department of Surgical Oncology, ${ }^{3}$ Department of Breast Surgical Oncology, ${ }^{4}$ The Sheikh \\ Khalifa Bin Zayed Al Nahyan Institute for Personalized Cancer Therapy, The University of Texas MD Anderson Cancer Center, Houston, TX, USA \\ Correspondence to: Funda Meric-Bernstam, MD. UT MD Anderson Cancer Center, 1400 Holcombe Boulevard, Unit 455, Houston, TX 77030, USA. \\ Email: fmeric@mdanderson.org. \\ Comment on: Kalinsky K, Sparano JA, Zhong X, et al. Pre-surgical trial of the AKT inhibitor MK-2206 in patients with operable invasive breast \\ cancer: a New York Cancer Consortium trial. Clin Transl Oncol 2018. [Epub ahead of print].
}

Submitted Sep 27, 2018. Accepted for publication Oct 10, 2018.

doi: $10.21037 / \mathrm{atm} .2018 .10 .32$

View this article at: http://dx.doi.org/10.21037/atm.2018.10.32

The course of drug discovery and development is expensive, and takes $10-15$ years with a high failure rate. Promising candidate drugs that pass in vitro investigations undergo in vivo testing for efficacy and safety. Novel cancer treatments are often assessed in patients who did not benefit from standard treatments. These heavily pretreated patients may have developed significant heterogeneity and multidrug resistance. They may also have a high tumor burden, which may limit activity of novel agents and stop their future development.

Animal studies provide evidence about drug effectiveness, however, preclinical pharmacokinetic (PK) and pharmacodynamic (PD) data determine how a new treatment might work in the clinic. Although, safety and tolerability is presumably established in phase I trials, there is often limited knowledge about biologic effects achieved at the recommended phase II dose. In a window of opportunity (WoO) trial, treatment naïve patients agree to postpone standard anticancer therapy for a short period, in order to receive the investigational agent in the period between the diagnostic biopsy and the delivery of the standard treatment (surgical resection, chemotherapy or radiation therapy), or as seen in this study, the trial takes advantage of natural scheduling delays that occur between initial consultation and surgery. This allows learning about anticancer activity of the intervention in a patient who is not exposed to previous therapies. Such studies can better define biological effects of the therapy and outline the target patient population for the following studies. In turn, development and identification of promising drugs could speed up.
WoO trials are usually short, in the order of 2-6 weeks of treatment, making it difficult to achieve clinical endpoints such as objective response. The endpoint of $\mathrm{WoO}$ trial is usually biomarker modulation. The collection of before and after treatment tumor biopsies allows determination of extent of target inhibition, cell proliferation and apoptosis, and identification of other biomarkers.

In breast cancer, deregulation and activation of the phosphoinositide 3-kinase (PI3K)/Akt pathway is associated with tumorigenesis and disease progression. Activating mutations in PIK3CA and $A K T 1$, and inactivating mutations in negative regulator PTEN are reported (1). In vitro data suggest that breast cancers with a low level PTEN expression or a mutant PIK3CA depend on Akt for oncogenic signaling (2). MK-2206 is an allosteric inhibitor of Akt that inhibits xenograft tumor growth in models with PIK3CA mutation or PTEN loss (3).

In a recent issue of Clinical and Translational Oncology, Kalinsky and colleagues reported results of a New York Cancer Consortium trial, an open-label, single-arm, presurgical WoO, multicenter trial with MK-2206 (4). Twelve newly diagnosed clinical stage I-III patients with histologically confirmed operable invasive breast cancer were enrolled. Formalin-fixed paraffin embedded samples were obtained from pretreatment core breast biopsies and posttreatment surgical specimens. Patients received two weekly doses of MK-2206 prior to surgery. The starting dose was $200 \mathrm{mg}$. Despite dose reductions, the trial was terminated after 12 patients due to grade III side effects observed at the lowest dose. In total, 5 patients experienced 
mucositis, 5 rash, 4 pruritus, and 3 hyperglycemia. There was a trend to reduction in pAkt after MK-2206 in both treatment and control groups. There was no significant change in expression of tumor markers Ki-67, pS6 Ser235/236, PTEN, and stathmin. There was no significant association between dose level and PK. Compared to controls, MK-2206 significantly increased serum glucose, insulin, C-peptide, and had a trend to an increase in IGFBP-3. C-reactive protein levels were increased in both MK-2206 and control arms, resulting in no difference. Seven patients had enough available tissue to perform mutation analysis and matched phospho-marker data. Three patients had PI3K/Akt pathway mutations: (I) PIK3CA H1047R; (II) PIK3CA E542K; and (III) PTEN. Because of the small cohort, it was not possible to evaluate differences in phospho-marker changes and PI3K pathway mutations. One limitation, also acknowledged by Kalinsky et al., was about the tumor collection. It is advised to perform pre and posttreatment sample collection with the same approach to limit the impact of tumor heterogeneity and to standardize the procedure (5).

WoO trials are trials that are more challenging to conduct, as often there is no clear clinical benefit for the patient due to the short treatment course. Thus, accrual relies on recruitment of truly informed patients that understand the importance of this study design for scientific advancement, and would like to "pay it forward". Thus, kudos to the New York Cancer Consortium for successfully conducts this multicenter trial. Equally notable is that fact that the team recognized that the side effects were greater than expected, and discontinued the trial.

WoO trials are usually conducted in an operable, curable population, thus they raise several concerns about safety and efficacy. While some trials are being conducted with well-established agents, many others are conducted with drugs earlier in development. At the minimum, the safety and recommended phase 2 dose should be established, and expected adverse need to be acceptable for an earlier, curable population.

In this trial, MK-2206 treatment was short, two doses were given in nine days, so that surgical resection would not be delayed. Primary endpoint was reduction of pAkt S473 in breast tumor tissue from diagnostic biopsy in surgery, which is a marker of target inhibition. Secondary endpoints included changes in other PI3K/Akt pathway markers, tumor proliferation (Ki-67), insulin growth factor pathway blood markers, pharmacokinetics (PK), genomics, and MK2206 tolerability. Primary and secondary aims were defined with an initial plan to enroll 24 patients. However, grade III side effects led to an early termination after enrolling only 12 patients. At the time of study initiation, the safety and tolerability of the compound had already been established in a phase I trial (6). A weekly $200 \mathrm{mg}$ dose was established as recommended phase II dose in a follow up study, at least in part based on toxicity profile (7). Notably in Kalinsky et al. the toxicities were what had been reported before, however the severity was greater than expected by investigators. This is in part potentially due to a lower tolerance for toxicity in a WoO setting by patients and physicians compared to trials conducted in heavily pretreated patients with more limited options. However, similar studies by other teams, $200 \mathrm{mg}$ dose was found to be too high and in a recent phase II trial, the weekly dose was defined as $75 \mathrm{mg}$ to keep the patients on study (8). Although early termination is not uncommon, a meta-analysis of $\mathrm{WoO}$ trials showed that treatment was stopped because of adverse effects in $4 \%$ of the patients (9). This emphasizes the delicate balance between scientific discovery and concerns about safety, especially in WoO setting.

In spite of its early termination, this report meaningfully adds to the literature. Although Gonzalez-Angulo et al. reported a significant decrease in pAkt S473 and pAkt T308 (10) with the combination of MK-2206 and paclitaxel by reverse phase protein arrays both in platelet rich plasma and in tumor biopsies, Ma et al. also described incomplete inhibition of Akt pathway (8) with combination of MK2206 and aromatase inhibition. Differences in drug doses, tissue preservation methods and assays (such as immunohistochemistry and reverse phase protein arrays) may have an effect on the results. Kalinsky et al. showed that there was a trend to decrease in pAkt after MK-2206 treatment, but this was also observed in the control group and there was no significant difference between these two groups. In Kalinsky et al. most of the assays did not detect a significant effect. The fact that trial accrual halted, limits study power. Enrolling 24 patients would allow having greater than $90 \%$ power to distinguish an effect. However, in the balance, at least at this dose the study suggests the safety concerns outweigh efficacy of the agent in this molecularly unselected patient population.

There are several WoO breast cancer trials ongoing, many testing endocrine agents and targeted therapies, metformin, statins, and anti-inflammatory drugs $(5,9,11)$. Short preoperative $\mathrm{WoO}$ studies teach us about molecular markers, mechanisms of action, clinical efficacy of novels agents and generate hypothesis for better treatment options, 
such as drug combinations. WoO trials will not replace but would be expected to help in designing better phase II/III studies by helping to identify predictive biomarkers and PD markers of response. In some cases, unfortunately the therapy may have adverse events that may not be deemed tolerable for this patient population. But in other rare occasions, like in the recent $\mathrm{WoO}$ trial with talazoparib in patients with deleterious $B R C A$ mutations reported by Litton et al. (12), there may be dramatic activity even with short term treatment, leading to a rapid path forward for a new therapeutic agent. Thus, biomarker-intensive $\mathrm{WoO}_{0}$ trials need to continue to be part of our armamentarium of clinical trials in early drug development.

\section{Acknowledgements}

None.

\section{Footnote}

Conflicts of Interest: F Meric-Bernstam reports receiving commercial research grants from and Novartis, AstraZeneca, Taiho, Genentech, Calithera, Debio International Group, Bayer, PUMA, Aileron, Jounce, CytoMx, Effector, Zymeworks, Curis, and Pfizer and is a consultant/advisory board member for Dialecta, Sumitomo Dainippon, Pieris Pharmaceuticals, Darwin Health, Samsung Bioepis, Aduro, and Spectrum, OrigiMed, Debiopharm International, Inflection Biosciences, Xencor, and Genentech. The other author has no conflicts of interest to declare.

\section{References}

1. Stemke-Hale K, Gonzalez-Angulo AM, Lluch A, et al. An integrative genomic and proteomic analysis of PIK3CA, PTEN, and AKT mutations in breast cancer. Cancer Res 2008;68:6084-91.

2. Saal LH, Johansson P, Holm K, et al. Poor prognosis in carcinoma is associated with a gene expression signature of aberrant PTEN tumor suppressor pathway activity. Proc

Cite this article as: Akcakanat A, Meric-Bernstam F. MK-2206 window of opportunity study in breast cancer. Ann Transl Med 2018;6(Suppl 1):S57. doi: 10.21037/atm.2018.10.32
Natl Acad Sci U S A 2007;104:7564-9.

3. Sangai T, Akcakanat A, Chen H, et al. Biomarkers of Response to Akt Inhibitor MK-2206 in Breast Cancer. Clin Cancer Res 2012;18:5816-28.

4. Kalinsky K, Sparano JA, Zhong X, et al. Pre-surgical trial of the AKT inhibitor MK-2206 in patients with operable invasive breast cancer: a New York Cancer Consortium trial. Clin Transl Oncol 2018. [Epub ahead of print].

5. Schmitz S, Duhoux F, Machiels JP. Window of opportunity studies: Do they fulfil our expectations? Cancer Treat Rev 2016;43:50-7.

6. Yap TA, Yan L, Patnaik A, et al. First-in-man clinical trial of the oral pan-AKT inhibitor MK-2206 in patients with advanced solid tumors. J Clin Oncol 2011;29:4688-95.

7. Yap TA, Yan L, Patnaik A, et al. Interrogating two schedules of the AKT inhibitor MK-2206 in patients with advanced solid tumors incorporating novel pharmacodynamic and functional imaging biomarkers. Clin Cancer Res 2014;20:5672-85.

8. Ma CX, Suman V, Goetz MP, et al. A Phase II Trial of Neoadjuvant MK-2206, an AKT Inhibitor, with Anastrozole in Clinical Stage II or III PIK3CA-Mutant ER-Positive and HER2-Negative Breast Cancer. Clin Cancer Res 2017;23:6823-32.

9. Marous M, Bieche I, Paoletti X, et al. Designs of preoperative biomarkers trials in oncology: a systematic review of the literature. Ann Oncol 2015;26:2419-28.

10. Gonzalez-Angulo AM, Krop I, Akcakanat A, et al. SU2C phase Ib study of paclitaxel and MK-2206 in advanced solid tumors and metastatic breast cancer. J Natl Cancer Inst 2015;107(3).

11. Maugeri-Sacca M, Barba M, Vici P, et al. Presurgical window of opportunity trial design as a platform for testing anticancer drugs: Pros, cons and a focus on breast cancer. Crit Rev Oncol Hematol 2016;106:132-42.

12. Litton JK, Scoggins M, Ramirez DL, et al. A feasibility study of neoadjuvant talazoparib for operable breast cancer patients with a germline BRCA mutation demonstrates marked activity. NPJ Breast Cancer 2017;3:49. 